TRANSACTIONS OF THE

AMERICAN MATHEMATICAL SOCIETY

Volume 357, Number 6, Pages 2119-2132

S 0002-9947(05)03870-5

Article electronically published on January 27, 2005

\title{
THE LIMIT SETS OF SCHOTTKY QUASICONFORMAL GROUPS ARE UNIFORMLY PERFECT
}

\author{
XIAOSHENG LI
}

\begin{abstract}
In this paper we study Schottky quasiconformal groups. We show that the limit sets of Schottky quasiconformal groups are uniformly perfect, and that the limit set of a given discrete non-elementary quasiconformal group has positive Hausdorff dimension.
\end{abstract}

\section{INTRODUCTION}

The notion of uniform perfectness first appeared in [10. A closed subset $A$ of the complex plane $\mathbb{C}$ is said to be uniformly perfect if there exists a constant $c>0$ such that

$$
A \cap\{z: c r \leq|z-a| \leq r\} \neq \varnothing,
$$

for any $a \in A$ and $0<r<\operatorname{diam}(A)$, where $\operatorname{diam}(A)$ is the Euclidean diameter of the set $A$. Pommerenke proved in [10] that a closed set $A \subset \mathbb{C}$ is uniformly perfect if, and only if, there is a constant $c>0$ such that

$$
\operatorname{cap}(A \cap\{|z-a| \leq r\}) \geq c r,
$$

for all $a \in A$ and $0<r<\operatorname{diam}(A)$, where cap, the logarithmic capacity of a compact set $E$, is defined by

$$
\operatorname{cap}(E)=\lim _{m \rightarrow \infty}\left(\max _{z_{1}, \ldots, z_{m} \in E} \prod_{\substack{\mu=1 \\ \mu \neq \nu}}^{m} \prod_{\substack{\nu=1 \\ \mu \neq \nu}}^{m} z_{\mu}-z_{\nu} \mid\right)^{\frac{1}{m(m-1)}} .
$$

The idea of uniform perfectness was first introduced in [1]. In that paper, Beardon and Pommerenke showed that the boundary of a domain $\Omega \subset \mathbb{C}$ not containing a neighborhood of infinity is uniformly perfect if, and only if, there exists a positive number $c$ such that

$$
\lambda_{\Omega}(z) \geq \frac{c}{\operatorname{dist}(z, \partial \Omega)},
$$

where $\lambda_{\Omega}(z)$ is the density of the Poincaré metric of $\Omega$, and dist $(z, \partial \Omega)$ is the Euclidean distance of $z$ to $\partial \Omega$. In the same paper, they also showed that the limit set $L(G)$ of a classical plane Schottky group $G$ is uniformly perfect.

Mañé and da Rocha [8] and Hinkkanen [6] proved independently that the Julia sets of rational functions are uniformly perfect.

In [7] Järvi and Vuorinen investigated the uniform perfectness of compact sets of $\overline{\mathbb{R}}^{n}$. They gave several equivalent conditions for uniform perfectness, and they

Received by the editors July 23, 2002.

2000 Mathematics Subject Classification. Primary 30C65.

Key words and phrases. Schottky quasiconformal group, uniformly perfect. 
showed that uniformly perfect sets have positive Hausdorff dimension and that the limit set of a non-elementary, finitely generated Kleinian group of $\overline{\mathbb{R}}^{n}$ is uniformly perfect.

In their 1987 paper [5], Gehring and Martin first introduced the notion of convergence groups in $\overline{\mathbb{R}}^{n}$. They studied the limit sets of discrete convergence groups and studied discrete quasiconformal groups, which are convergence groups. Tukia, in 11, extended the notion of convergence groups to compact Hausdorff spaces satisfying the first axiom of countability and investigated convergence groups in that setting.

Petra Bonfert-Taylor and Edward C. Taylor in 2] and 3] extended the PattersonSullivan theory to the quasiconformal group setting. They investigated the connection between the exponent of convergence of Poincaré series and the Hausdorff dimension of the limit sets of quasiconformal groups. They showed that the Hausdorff dimension of the conical limit set $L_{c}(G)$ has an upper bound; see Corollary 1.6. and Conjecture 1.7. in [3].

Our main results are the following theorems.

1.1. Theorem. For an integer $t$ with $t \geq 2$, let $G=\left\langle g_{1}, g_{2}, \ldots, g_{t}\right\rangle$ be a Schottky $K$-quasiconformal group. Then the limit set $L(G)$ is a uniformly perfect set.

1.2. Theorem. Let $G$ be a discrete non-elementary quasiconformal group. Then the Hausdorff dimension of the limit set $L(G)$ is positive.

This paragraph is about the organization of this paper. Section 2 contains some notations and basic definitions. We talk about moduli of curve families, and basic properties of $K$-quasiconformal mappings in $\overline{\mathbb{R}}^{n}$, then we recall some results about convergence groups and their limit sets, which are needed in this paper. In section 3 , the notion of a Schottky quasiconformal group is introduced, and we give representations of the limit set $L(G)$ and the regular set $\Omega(G)$. In section 4 , we show that the limit set $L(G)$ of a Schottky quasiconformal group is uniformly perfect. In section 5 , we generalize our theorem in section 4 , and we show that if $G$ is a

discrete non-elementary quasiconformal group, then the Hausdorff dimension of the limit set $L(G)$ is positive.

\section{Preliminaries}

Let $\mathbb{R}^{n}$ be the $n$-dimensional Euclidean space and let $\overline{\mathbb{R}}^{n}$ be the one-point compactification of $\mathbb{R}^{n}$. We write $e_{1}, e_{2}, \ldots, e_{n}$ for the standard basis of $\mathbb{R}^{n}$, where $e_{1}=(1,0, \ldots, 0)$, etc. For $x \in \mathbb{R}^{n}$, we define

$$
|x|=\sqrt{x_{1}^{2}+x_{2}^{2}+\ldots+x_{n}^{2}} .
$$

We write $B^{n}(x, r)$ for the open ball centered at $x$ with radius $r$, and write $S^{n-1}(x, r)=\partial B^{n}(x, r)$ for the boundary of $B^{n}(x, r)$.

The stereographic projection $\pi: \overline{\mathbb{R}}^{n} \rightarrow S^{n}\left(\frac{1}{2} e_{n+1}, \frac{1}{2}\right)$ is given by

$$
\pi(x)=e_{n+1}+\frac{x-e_{n+1}}{\left|x-e_{n+1}\right|^{2}} .
$$

Clearly, for $x=\left(x_{1}, x_{2}, \ldots, x_{n}\right) \in \mathbb{R}^{n}$, we have

$$
\pi(x)=\left(\frac{x_{1}}{|x|^{2}+1}, \ldots, \frac{x_{n}}{|x|^{2}+1}, \frac{|x|^{2}}{|x|^{2}+1}\right),
$$


and we define $\pi(\infty)=e_{n+1}$. The function $\pi: \overline{\mathbb{R}}^{n} \rightarrow S^{n}\left(\frac{1}{2} e_{n+1}, \frac{1}{2}\right)$ is a bijective map. We define the chordal metric on $\overline{\mathbb{R}}^{n}$ by $q(x, y)=|\pi(x)-\pi(y)|$ for $x, y \in \overline{\mathbb{R}}^{n}$. For subsets $X, Y \subset \overline{\mathbb{R}}^{n}$, the diameter of $X$ is defined as $q(X)=\sup _{x, y \in X} q(x, y)$ and the distance between $X$ and $Y$ is given by $q(X, Y)=\inf _{x \in X, y \in Y} q(x, y)$.

Let $B_{q}(x, r)$ denote the chordal ball

$$
B_{q}(x, r)=\left\{y \in \overline{\mathbb{R}}^{n}: q(x, y)<r\right\} .
$$

A Möbius transformation acting on $\overline{\mathbb{R}}^{n}$ is a finite composition of reflections in spheres and hyperplanes. We write $G M\left(\overline{\mathbb{R}}^{n}\right)$ for the group of all Möbius transformations. A Möbius group is a subgroup of $G M\left(\overline{\mathbb{R}}^{n}\right)$. Let $D$ and $D^{\prime}$ be domains in $\overline{\mathbb{R}}^{n}$. A homeomorphism $f: D \rightarrow D^{\prime}$ is a conformal mapping if $f \in C^{1}$, and

$$
\left|f^{\prime}(x) h\right|=\max _{|s|=1}\left|f^{\prime}(x) s\right||h|
$$

for any $x \in D$ and $h \in \mathbb{R}^{n}$. If $D$ and $D^{\prime}$ are domains in $\overline{\mathbb{R}}^{n}$, then a homeomorphism $f: D \rightarrow D^{\prime}$ is conformal if $f$ is conformal in $D \backslash\left\{\infty, f^{-1}(\infty)\right\}$. Möbius transformations are conformal mappings. By Liouville's theorem, when $n \geq 3$, the only conformal mappings in $\overline{\mathbb{R}}^{n}$ are Möbius transformations.

Let $\Gamma$ be a family of curves in $\overline{\mathbb{R}}^{n}$, and let $\mathcal{F}(\Gamma)$ be the set of admissible functions, i.e., non-negative Borel functions $\rho: \overline{\mathbb{R}}^{n} \rightarrow \mathbb{R} \cup\{\infty\}$ such that

$$
\int_{\gamma} \rho d s \geq 1
$$

for any locally rectifiable $\gamma \in \Gamma$. For $p \geq 1$ the $p$-modulus of $\Gamma$ is defined as

$$
M_{p}(\Gamma)=\inf _{\rho \in \mathcal{F}(\Gamma)} \int_{\mathbb{R}^{n}} \rho^{p} d m .
$$

The $p$-modulus $M_{p}$ is an outer measure in the space of all curve families in $\overline{\mathbb{R}}^{n}$. When $p=n$ we denote $M_{p}(\Gamma)$ by $M(\Gamma)$. The quantity $M(\Gamma)$ is conformally invariant, i.e., if $f: D \rightarrow D^{\prime}$ is a conformal mapping and $\Gamma$ is a curve family in $D$, then we have

$$
M(f \Gamma)=M(\Gamma),
$$

where $f \Gamma=\{f \circ \gamma: \gamma \in \Gamma\}$. From now on we denote $f \Gamma$ by $\Gamma^{\prime}$.

2.1. Definition. Let $f: D \rightarrow D^{\prime}$ be a homeomorphism. The inner and outer dilatations $K_{I}(f)$ and $K_{O}(f)$ of the mapping $f$ are defined by

$$
K_{I}(f)=\sup \frac{M\left(\Gamma^{\prime}\right)}{M(\Gamma)}, \quad K_{O}(f)=\sup \frac{M(\Gamma)}{M\left(\Gamma^{\prime}\right)},
$$

where the suprema are taken over all curve families $\Gamma$ in $D$ such that $M(\Gamma)$ and $M\left(\Gamma^{\prime}\right)$ are not simultaneously zero or $\infty$. The maximal dilatation of the mapping $f$ is defined as $K(f)=\max \left\{K_{I}(f), K_{O}(f)\right\}$. The mapping $f$ is said to be $K$-quasiconformal if $K(f) \leq K<\infty$, for some constant $K$. Equivalently, the mapping $f$ is $K$-quasiconformal if, and only if,

$$
\frac{M(\Gamma)}{K} \leq M\left(\Gamma^{\prime}\right) \leq K M(\Gamma)
$$

for every curve family $\Gamma$ in $D$. 
Let $f$ be $K_{1}$-quasiconformal and let $g$ be $K_{2}$-quasiconformal. Then the inverse function $f^{-1}$ is $K_{1}$-quasiconformal, and $f \circ g$ is $K_{1} K_{2}$-quasiconformal.

Let $G$ be a group of self-homeomorphisms of a domain $D$. We say that the group $G$ is a $K$-quasiconformal group if every element of $G$ is a $K$-quasiconformal selfmapping of $D$. We say that $G$ is a quasiconformal group if it is a $K$-quasiconformal group for some constant $K$. Möbius groups are 1-quasiconformal groups.

2.2. Definition (Gehring and Martin). A group $G$ of self-homeomorphisms of $\overline{\mathbb{R}}^{n}$ is said to be a convergence group if each infinite subfamily of $G$ has a sequence $\left\{g_{i}\right\}$ of distinct elements such that one of the following is true:

(1) There exists a self-homeomorphism $g$ of $\overline{\mathbb{R}}^{n}$ with the property that

$$
\lim _{i \rightarrow \infty} g_{i}=g, \quad \lim _{i \rightarrow \infty} g_{i}^{-1}=g^{-1}
$$

uniformly on $\overline{\mathbb{R}}^{n}$.

(2) There exist $x_{0}$ and $y_{0}$ in $\overline{\mathbb{R}}^{n}$ (possibly $x_{0}=y_{0}$ ) with the property that

$$
\lim _{i \rightarrow \infty} g_{i}=y_{0}, \quad \lim _{i \rightarrow \infty} g_{i}^{-1}=x_{0}
$$

uniformly on compact subsets of $\overline{\mathbb{R}}^{n} \backslash\left\{x_{0}\right\}$ and $\overline{\mathbb{R}}^{n} \backslash\left\{y_{0}\right\}$, respectively, or simply, c-uniformly in $\overline{\mathbb{R}}^{n} \backslash\left\{x_{0}\right\}$ and $\overline{\mathbb{R}}^{n} \backslash\left\{y_{0}\right\}$, respectively.

In the above definition, the points $y_{0}$ and $x_{0}$ are called the attracting and repelling points of $\left\{g_{i}\right\}$, respectively. Both points are called limit points of $G$. Also the group $G$ is said to be discrete if (1) never happens, and the group $G$ is said to be precompact if (1) always happens. By Väisälä [12], Gehring and Martin [5] quasiconformal groups are convergence groups, and so are Möbius groups.

Let $G$ be a group of self-homeomorphisms of a domain $D$ in $\overline{\mathbb{R}}^{n}$. The group $G$ is said to be discontinuous at $x \in D$ if there is a neighborhood $U$ of $x$ in $D$ such that $g(U) \cap U=\varnothing$ for all but finitely many $g \in G$. We say that the group $G$ is discontinuous if $G$ is discontinuous at some point $x \in D$. The group $G$ is said to be properly discontinuous in an open set $O \subset D$ if for each compact set $F \subset O$, we have $g(F) \cap F=\varnothing$ for all but finitely many $g \in G$. We say that the group $G$ is discrete if there is no sequence of distinct elements $\left\{g_{n}\right\} \subset G$ such that $g_{n} \rightarrow g$ uniformly on compact subsets of $D$, or c-uniformly in $D$, where $g$ is a selfhomeomorphism of $D$. Let $G$ be a convergence group of self-homeomorphisms of the domain $D$. The regular set of $G$, denoted by $\Omega(G)$, is given by $\Omega(G)=\{x \in D$ : $G$ is discontinuous at $x\}$, and the limit set $L(G)$ is defined by $L(G)=\bar{D} \backslash \Omega(G)$. Let $X$ be a subset of $\overline{\mathbb{R}}^{n}$. We say that the set $X$ is $G$-invariant if $f(X)=X$ for every $f \in G$. If the number of elements of $L(G)$ is less than or equal to two, we say that $G$ is an elementary convergence group. Otherwise $G$ is called a non-elementary convergence group.

2.3. Theorem (Gehring and Martin). Let $G$ be a convergence group in $\overline{\mathbb{R}}^{n}$. Let $\Omega(G)$ and $L(G)$ be the regular set and the limit set of $G$, respectively. The following statements hold:

(1) The set $\Omega(G)$ is open, and the set $L(G)$ is closed.

(2) The intersection $\Omega(G) \cap L(G)$ is the empty set.

(3) The union $\Omega(G) \cup L(G)$ is $\overline{\mathbb{R}}^{n}$.

(4) Both $\Omega(G)$ and $L(G)$ are $G$-invariant. 
Let $G$ be a convergence group. By Gehring and Martin [5], and Tukia [11], an element $g \in G$ is elliptic if the group generated by $g$ is precompact. For a nonelliptic element $g \in G$ we say that $g$ is parabolic if the number of the fixed points of $g$ is one, and $g$ is loxodromic if the number of the fixed points of $g$ is two. A non-elliptic element $g \in G$ can fix at most two points.

2.4. Theorem (Tukia). If $G$ is a non-elementary convergence group, then the limit set $L(G)$ is an infinite perfect set, and $L(G)$ is in the accumulation set of any orbit $G x=\{f(x): f \in G\}$, for $x \in D$, and thus if $x \in L(G)$, we have

$$
\overline{G x}=L(G) \text {. }
$$

See also 5 .

Let $E, F, D$ be subsets of $\overline{\mathbb{R}}^{n}$. We use $\Delta(E, F ; D)$ to denote the family of all closed paths joining $E$ and $F$ in $D$; see $\left[12\right.$ and $\left[13\right.$. Let $D \subset \overline{\mathbb{R}}^{n}$ be a domain and let $E$ be a compact subset of $D$. Then we call the pair $(D, E)$ a condenser and the capacity of the condenser is $\operatorname{cap}(D, E)=M(\Delta(E, \partial D ; D))$. A ring domain $D$ is a domain such that the complement of $D$ has exactly two components $E$ and $F$. In this case, we write $D=R(E, F)$. The modulus of $D$ is given by

$$
\bmod (D)=\left(\frac{M\left(\Delta\left(E, F ; \overline{\mathbb{R}}^{n}\right)\right)}{\omega_{n-1}}\right)^{\frac{1}{1-n}}
$$

where $\omega_{n-1}>0$ is the $(n-1)$-dimensional measure of $S^{n-1}$.

The proof of the following lemma can be found in [13].

2.5. Lemma. For two numbers $r$ and $s$ with $0<r<s<1$ and $x \in \overline{\mathbb{R}}^{n}$, the domain $D=B_{q}^{n}(x, s) \backslash \bar{B}_{q}^{n}(x, r)$ is called a chordal ring. Then the modulus of the chordal ring $D$ is given by

$$
\bmod (D)=\log \left(\frac{s \sqrt{1-r^{2}}}{r \sqrt{1-s^{2}}}\right) .
$$

A ring domain $D$ is called a Teichmüller ring if the complementary components of $D$ are $\left[-e_{1}, 0\right]$ and $\left[s e_{1}, \infty\right]$, where $s>0$. We use $\tau_{n}(s)$ to denote the modulus of the family of all curves connecting the two complementary components of $D$, and we also briefly write $\tau(s)$ instead of $\tau_{n}(s)$. The function $\tau(s)$ is decreasing and

$$
\lim _{s \rightarrow 0^{+}} \tau(s)=\infty, \quad \lim _{s \rightarrow \infty} \tau(s)=0 .
$$

Let $X$ be a subset of $\overline{\mathbb{R}}^{n}$ and let $D=R(E, F)$ be a ring domain. Then we say that $D$ separates $X$ if $X \cap D=\varnothing, X \cap E \neq \varnothing$ and $X \cap F \neq \varnothing$. Let $X$ and $Y$ be two subsets of $\overline{\mathbb{R}}^{n}$. We say that the ring domain $D=R(E, F)$ separates the sets $X$ and $Y$ if we have $X \subset E, Y \subset F$ or $Y \subset E, X \subset F$.

2.6. Definition. Let $X \subset \overline{\mathbb{R}}^{n}$ be a closed subset containing at least two points. Then we say that $X$ is a uniformly perfect set if there exists a constant $C$ such that for any ring domain $D \subset \overline{\mathbb{R}}^{n}$ separating $X$, the modulus of $D$ is bounded above by the constant $C$.

The following two lemmas are due to Järvi and Vuorinen; see [7].

2.7. Lemma. Let $X$ be a closed subset of $\overline{\mathbb{R}}^{n}$ containing at least two points. Then $X$ is uniformly perfect if, and only if, there is a constant $C$ such that the modulus of any chordal ring domain separating $X$ is bounded above by the constant $C$. 
2.8. Lemma. Let $D=R(E, F)$ be a ring domain in $\overline{\mathbb{R}}^{n}$. Then the following inequality is true:

$$
\operatorname{cap}(D) \geq 2^{1-n} \tau\left(\frac{2 q(E, F)}{\min \{q(E), q(F)\}}\right)
$$

where $\tau$ is the Teichmüller ring capacity.

\section{SCHOTTKY QUASICONFORMAL GROUPS}

For $t \geq 2$ let $B_{1}, B_{2}, \ldots, B_{2 t-1}$ and $B_{2 t}$ be $t$ pairs of open balls with disjoint closures in $\overline{\mathbb{R}}^{n}$. Set $C_{1}=\partial B_{1}, C_{2}=\partial B_{2}, \ldots, C_{2 t-1}=\partial B_{2 t-1}$ and $C_{2 t}=\partial B_{2 t}$. The classical Schottky group is a Möbius group $\Gamma$ such that $\Gamma$ is finitely generated by $t$ Möbius transformations $g_{1}, g_{2}, \ldots, g_{t}$ of $\overline{\mathbb{R}}^{n}$ with the property that

$$
g_{i}\left(B_{2 i-1}\right)=\overline{\mathbb{R}}^{n} \backslash \bar{B}_{2 i}
$$

for all $i$ with $1 \leq i \leq t$.

3.1. Theorem. Let $\Gamma$ be a classical Schottky group defined as above. Then $\Gamma$ is a free group and every element of $\Gamma$ is loxodromic. In addition, the group $\Gamma$ is a discrete Möbius group.

See [4] and 9 .

Discrete Möbius groups are also called Kleinian groups. In the rest of this section, we assume that $B_{1}, B_{2}, \ldots, B_{2 t-1}$ and $B_{2 t}$ are open quasiballs with disjoint closures, i.e., for each $i$ with $1 \leq i \leq 2 t$, the set $B_{i}$ is the image of a ball in $\overline{\mathbb{R}}^{n}$ under some quasiconformal mapping of $\overline{\mathbb{R}}^{n}$. For $1 \leq i \leq 2 t$, we set $C_{i}=\partial B_{i}$.

3.2. Definition. A group $G=\left\langle g_{1}, g_{2}, \ldots, g_{t}\right\rangle$ is called a Schottky quasiconformal group acting on $\overline{\mathbb{R}}^{n}$ if $G$ is $K$-quasiconformal group on $\overline{\mathbb{R}}^{n}$ for some $K \geq 1$, and

$$
g_{i}\left(B_{2 i-1}\right)=\overline{\mathbb{R}}^{n} \backslash \bar{B}_{2 i}
$$

for all $i$ between 1 and $t$.

We give an example of a Schottky quasiconformal group. Let $\Gamma$ be a classical Schottky group. Let $g$ be a $K$-quasiconformal mapping on $\overline{\mathbb{R}}^{n}$. Then the conjugate $g \circ \Gamma \circ g^{-1}$ of $\Gamma$ under $g$ is a Schottky $K^{2}$-quasiconformal group.

Notation. Set $A_{1}=\bigcup_{i=1}^{2 t} \overline{B_{i}}, D_{1}=\overline{\mathbb{R}}^{n} \backslash A_{1}$. We use $\Sigma$ to denote $D_{1}$ throughout the remainder of this paper. Clearly the set $\Sigma$ is open.

3.3. Lemma. The Schottky quasiconformal group $G=\left\langle g_{1}, g_{2}, \ldots, g_{t}\right\rangle$ is a discontinuous group, and hence it is a discrete group.

Notation. Consider $B_{1}$; there are $2 t-1$ smaller quasiballs inside $B_{1}$, which are images of $B_{1}, B_{3}, B_{4}, \ldots, B_{2 t}$ under the $K$-quasiconformal mapping $g_{1}^{-1}$. Similarly, for each of the remaining $B_{i}$, there are $2 t-1$ smaller quasiballs inside the $B_{i}$ which are the images of certain $2 t-1$ original quasiballs under $g_{j}$ or $g_{j}^{-1}$ for some $j$ between 1 and $t$. Clearly, there are a total of $2 t(2 t-1)$ such smaller quasiballs, called the second generation quasiballs of the group $G$. For $i$ with $1 \leq i \leq 2 t(2 t-1)$, we denote these quasiballs by $B_{i}^{2}$, and set

$$
A_{2}=\bigcup_{i=1}^{2 t(2 t-1)} \overline{B_{i}^{2}} .
$$


The complement of $A_{2}$ is denoted by

$$
D_{2}=\overline{\mathbb{R}}^{n} \backslash A_{2} .
$$

The set $A_{2}$ is closed and $D_{2}$ is open.

Similarly, we can define $A_{p}$ as the closure of the union of all $p$-th generation open quasiballs, and $D_{p}$ is the complement of $A_{p}$. The set $A_{p}$ is closed and $D_{p}$ is open. Also we have

$$
A_{1} \supset A_{2} \supset \cdots \supset A_{p} \supset \cdots
$$

and

$$
\Sigma \subset D_{2} \subset \cdots \subset D_{p} \subset \cdots .
$$

3.4. Definition. Let $G=\left\langle g_{1}, g_{2}, \ldots, g_{t}\right\rangle$ be a Schottky quasiconformal group. We know that $G$ is a free group. For any non-identity $g \in G, g$ can be written uniquely as

$$
g=\prod_{i=1}^{q} g_{t_{i}}^{s_{i}},
$$

where $g_{t_{i}} \in\left\{g_{1}, g_{2}, \ldots, g_{t}\right\}$ and $g_{t_{i}} \neq g_{t_{i+1}}$ for $1 \leq i \leq q-1$, each $s_{i}$ is an integer with $s_{i} \neq 0$ for $1 \leq i \leq q$, for some positive integer $q$. We define the index of $g$ by

$$
\text { ind }(g)=\sum_{i=1}^{q}\left|s_{i}\right|,
$$

for $g \neq I$. We define ind $(I)=0$.

3.5. Lemma. Let $G$ be a Schottky quasiconformal group. For $m \geq 1$, the sets $A_{m}$ and $D_{m}$ are defined as before. Then we have the following:

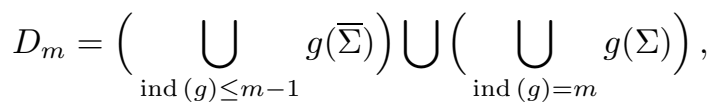

and the complement of $D_{m}$ is given by

$$
A_{m}=\bigcup\left\{g\left(\overline{B_{i}}\right): \text { ind }(g)=m, 1 \leq i \leq 2 t, g\left(\overline{B_{i}}\right) \subset A_{1}\right\},
$$

where the set $A_{1}=\bigcup_{i=1}^{2 t} \overline{B_{i}}$.

In addition, if two mappings $f$ and $g$ are in the group $G$, and $f \neq g$, then

$$
f(\Sigma) \cap g(\Sigma)=\varnothing .
$$

If two mappings $f$ and $g$ are in the group $G$ with $f \neq g$, and ind $(f)=\operatorname{ind}(g)$, then

$$
f\left(\overline{B_{i}}\right) \cap g\left(\overline{B_{i}}\right)=\varnothing,
$$

when $f\left(\overline{B_{i}}\right)$ and $g\left(\overline{B_{i}}\right)$ are both inside the set $A_{1}$.

Proof. It is easy to check this by induction. We omit the proof.

3.6. Lemma. Let $G=\left\langle g_{1}, g_{2}, \ldots, g_{t}\right\rangle$ be the Schottky quasiconformal group defined as above, and for any integer $p \geq 1$, let $A_{p}$ be the closure of the union of all the $p$-th generation open quasiballs. We define

$$
d_{p}=\max _{E \subset A_{p}} q(E),
$$

where the maximum is taken over all components $E$ of $A_{p}$. Then the limit of the sequence $\left\{d_{p}\right\}$ exists, and in fact, the limit is zero. 
Proof. Clearly $\left\{d_{p}\right\}$ is a decreasing sequence and so it has a limit $d$. We suppose that $d>0$. By the Cantor diagonal process, we can choose a sequence of sets $\left\{\Delta_{p}\right\}$ with $\Delta_{p}$ a component of $A_{p}$ such that the following hold:

$$
\Delta_{i} \supset \Delta_{i+1}
$$

for $i$ with $1 \leq i<\infty$, and

$$
\lim _{p \rightarrow \infty} q\left(\Delta_{p}\right)=d
$$

For each set $\Delta_{p}$ with $1 \leq p<\infty$, there exists a quasiconformal mapping $f_{p} \in G$ such that

$$
\Delta_{p}=f_{p}\left(\overline{\mathbb{R}}^{n} \backslash B_{j_{p}}\right)
$$

for some $j_{p}$ with $1 \leq j_{p} \leq 2 t$. Since there are only a finite number of $B_{j_{p}}$ and there are infinitely many $f_{p} \in G$, there exists at least one $B_{j_{p}}$, denoted by $B_{0}^{\prime}$, such that

$$
\Delta_{p}=f_{p}\left(\overline{\mathbb{R}}^{n} \backslash B_{0}^{\prime}\right)
$$

for infinitely many $f_{p} \in G$. Without loss of generality, we assume that (3) is true for all $p$ with $1 \leq p<\infty$.

On the other hand, the group $G$ is a discrete convergence group. For the sequence $\left\{f_{p}\right\}$ of distinct elements of $G$, there exists a subsequence of $\left\{f_{p}\right\}$ which is still denoted by $\left\{f_{p}\right\}$ without danger of confusion, and there exist two points $x_{0}^{\prime}, y_{0}^{\prime} \in \overline{\mathbb{R}}^{n}$ such that

$$
\lim _{p \rightarrow \infty} f_{p}=y_{0}^{\prime}, \quad \lim _{p \rightarrow \infty} f_{p}^{-1}=x_{0}^{\prime}
$$

uniformly on compact subsets of $\overline{\mathbb{R}}^{n} \backslash\left\{x_{0}^{\prime}\right\}$ and $\overline{\mathbb{R}}^{n} \backslash\left\{y_{0}^{\prime}\right\}$, respectively. But this contradicts (3), and we complete our proof for this lemma.

3.7. Theorem. Let $G=\left\langle g_{1}, g_{2}, \ldots, g_{t}\right\rangle$ be a Schottky quasiconformal group. For each $i$ with $i \geq 1$, let $A_{i}$ be the closure of the union of all the $i$-th generation open quasiballs and let $D_{i}$ be the complement of $A_{i}$ with respect to $\overline{\mathbb{R}}^{n}$. Then the limit set $L(G)$ is given by

$$
L(G)=\bigcap_{i=1}^{\infty} A_{i},
$$

and the regular set $\Omega(G)$ is given by

$$
\Omega(G)=\bigcup_{i=1}^{\infty} D_{i} .
$$

The proof is easy and we omit it.

\section{UNIFORM PERFECTNESS OF $L(G)$}

In this section, we show that the limit set of a Schottky quasiconformal group is uniformly perfect, and by a result of Järvi and Vuorinen [7] the limit set then has positive Hausdorff dimension.

In the following, we denote by $U(x, r, R)$ a chordal ring centered at $x$ with radii $r$ and $R$. 
Proof of Theorem 1.1. We suppose that the limit set $L(G)$ is not uniformly perfect. Then there exists a sequence of distinct chordal rings $U_{m}\left(x_{m}, r_{m}, R_{m}\right)$ separating $L(G)$ with $0<r_{m}<R_{m}<1$, such that

$$
\bmod \left(U_{m}\left(x_{m}, r_{m}, R_{m}\right)\right) \rightarrow \infty
$$

For simplicity, we write $U_{m}=U_{m}\left(x_{m}, r_{m}, R_{m}\right)$ and $V_{m}=B_{q}\left(x_{m}, r_{m}\right)$, where $B_{q}\left(x_{m}, r_{m}\right)$ is a chordal ball centered at $x_{m}$ with radius $r_{m}$. Without loss of generality, we may assume that $x_{m} \rightarrow x_{0}$ for some $x_{0} \in \overline{\mathbb{R}}^{n}$. Since $L(G)$ is perfect, we have

$$
\lim _{m \rightarrow \infty} r_{m}=\lim _{m \rightarrow \infty} R_{m}=0 .
$$

By Theorem 3.7. $L(G)=\bigcap_{m=1}^{\infty} A_{m}$, where $A_{m}$ is the closure of the union of all the $m$-th generation quasiballs. Let $m_{0}$ be a fixed positive integer. Then there is one component of $A_{m_{0}}$ which contains $x_{0}$, and we denote by $\Delta_{m_{0}}^{\prime}$ the interior of that component. Since $R_{m} \rightarrow 0$ there exists a positive integer $M_{1}$ such that when $m \geq M_{1}$, we have $U_{m} \subset \Delta_{m_{0}}^{\prime}$. Pick a fixed integer $m_{1}$ with $m_{1} \geq M_{1}$; then we have

$$
U_{m_{1}} \subset \Delta_{m_{0}}^{\prime}
$$

Note that the chordal ring domain $U_{m_{1}}$ separates $L(G)$. Thus there exists a positive integer $M_{2}>m_{0}$ such that whenever $m \geq M_{2}$, the set $U_{m_{1}}$ separates the set $A_{m}$.

Pick an integer $m_{2}$ with $m_{2} \geq M_{2}$; then $U_{m_{1}}$ separates $A_{m_{2}}$. In particular, then $U_{m_{1}} \cap A_{m_{2}}=\varnothing$. Clearly, we have $m_{2}>m_{0}$.

Now consider the set $\Delta_{m_{0}}^{\prime} \cap L(G)$. If $\Delta_{m_{0}}^{\prime} \cap L(G) \subset V_{m_{1}}$, where $V_{m_{1}}$ is the chordal ball centered at $x_{m_{1}}$ with radius $r_{m_{1}}$, then there exists a $K$-quasiconformal mapping $f \in G$ such that

$$
f^{-1}\left(\Delta_{m_{0}}^{\prime} \backslash A_{m_{0}+1}\right)=\Sigma
$$

and therefore the ring domain $f^{-1}\left(U_{m_{1}}\right)$ separates some component $B_{j}$ of the interior of $A_{1}$ and $\left(A_{1} \backslash \overline{B_{j}}\right) \cap L(G)$. We write $f^{-1}\left(U_{m_{1}}\right)=R(E, F)$, where $E \supset B_{j}$ and $F \supset\left(A_{1} \backslash \overline{B_{j}}\right) \cap L(G)$. Then we have

$$
\min \{q(E), q(F)\} \geq \min _{1 \leq i \leq 2 t}\left\{q\left(B_{i} \cap L(G)\right)\right\}>0 .
$$

Note also that

$$
q(E, F) \leq \max _{1 \leq j \leq 2 t}\left\{q\left(B_{j},\left(A_{1} \backslash \overline{B_{j}}\right) \cap L(G)\right)\right\}<\infty .
$$

Thus we have

$$
\operatorname{cap}\left(U_{m_{1}}\right) \geq \frac{2^{1-n}}{K} \tau\left(\frac{2 \max _{1 \leq j \leq 2 t}\left\{q\left(B_{j},\left(A_{1} \backslash \overline{B_{j}}\right) \cap L(G)\right)\right\}}{\min _{1 \leq i \leq 2 t}\left\{q\left(B_{i} \cap L(G)\right)\right\}}\right)>0,
$$

and this is a contradiction.

From now on we suppose that the chordal ring domain $U_{m_{1}}$ separates $\Delta_{m_{0}}^{\prime} \cap L(G)$.

First for $i$ with $m_{0} \leq i \leq m_{2}-1$ let us consider the set $A_{i}$. There are finitely many components of the set $\Delta_{m_{0}}^{\prime} \cap A_{i}$. Now we need to use some ad hoc definitions. For each component $\Delta$ of the interior of $\Delta_{m_{0}}^{\prime} \cap A_{i}$, we call $\Delta \cap L(G)$ an effective set of $\Delta_{m_{0}}^{\prime} \cap A_{i}$.

We define, for $i$ with $m_{0} \leq i \leq m_{2}-1$, that

$$
\Theta_{i}=\left\{\Delta \cap L(G): \Delta \text { is a component of the interior of } \Delta_{m_{0}}^{\prime} \cap A_{i}\right\},
$$


i.e., $\Theta_{i}$ is the set of all the effective sets of $\Delta_{m_{0}}^{\prime} \cap A_{i}$. Clearly the number of the elements of the set $\Theta_{m_{0}+1}$ is given by

$$
\# \Theta_{m_{0}+1}=2 t-1 \text {. }
$$

Here and later we use the symbol \# to denote the number of elements in a set.

In general, for $i$ between $m_{0}+1$ and $m_{2}-1$, the number of the elements of $\Theta_{i}$ is given by

$$
\# \Theta_{i}=(2 t-1)^{i-m_{0}},
$$

and clearly, $\# \Theta_{m_{0}}=1$.

For a given effective set $E_{1} \in \Theta_{i}$, an effective set $F_{1} \in \Theta_{i}$ is said to be a close relative set of $E_{1}$ if $F_{1}$ and $E_{1}$ are inside the same element of the set $\Theta_{i-1}$ as point sets.

In the following, we proceed in two steps.

(1) Let us pick an effective set $E_{1}$ from $\Theta_{m_{2}-1}$, and consider all the close relative sets of $E_{1}$. We denote by $E_{1}^{\prime}$ the set of all the close relative sets of $E_{1}$, together with the set $E_{1}$.

If there exist two effective sets $\Delta_{1} \cap L(G)$ and $\Delta_{2} \cap L(G)$ in the set $E_{1}^{\prime}$ such that the chordal ring domain $U_{m_{1}}$ separates the two sets $\Delta_{1} \cap L(G)$ and $\Delta_{2} \cap L(G)$, where $\Delta_{1}$ and $\Delta_{2}$ are two components of the interior of $A_{m_{2}-1}$ which are inside $\Delta^{\prime}$, one component of the interior of $A_{m_{2}-2}$, then there exists a $K$-quasiconformal mapping $f \in G$ with

$$
f^{-1}\left(\Delta^{\prime} \backslash A_{m_{2}-1}\right)=\Sigma
$$

Note that the ring domain $f^{-1}\left(U_{m_{1}}\right)$ separates two sets $B_{i} \cap L(G)$ and $B_{j} \cap$ $L(G)$, where $B_{i}$ and $B_{j}$ are two distinct components of the set $\overline{\mathbb{R}}^{n} \backslash \bar{\Sigma}$. We write $f^{-1}\left(U_{m_{1}}\right)=R(E, F)$. Therefore we have

$$
\min \{q(E), q(F)\} \geq \min _{1 \leq i \leq 2 t}\left\{q\left(B_{i} \cap L(G)\right)\right\}>0 .
$$

In addition, the following is true:

$$
q(E, F) \leq \max _{1 \leq i, j \leq 2 t}\left\{q\left(B_{i} \cap L(G), B_{j} \cap L(G)\right)\right\}<\infty
$$

and therefore,

$$
\operatorname{cap}\left(U_{m_{1}}\right) \geq \frac{2^{1-n}}{K} \tau\left(\frac{2 \max _{1 \leq i, j \leq 2 t}\left\{q\left(B_{i} \cap L(G), B_{j} \cap L(G)\right)\right\}}{\min _{1 \leq i \leq 2 t}\left\{q\left(B_{i} \cap L(G)\right)\right\}}\right)>0,
$$

but this is a contradiction since $m_{1}$ is arbitrarily chosen.

If there are no two effective sets in $E_{1}^{\prime}$ which are separated by the chordal ring domain $U_{q_{m_{1}}}$, we consider all the remaining effective sets in $\Theta_{m_{2}-1}$, and repeat the above process.

Now let us go through the second step.

(2) We assume that there are no two effective sets in $\Theta_{m_{2}-1}$, which are close relative sets to each other, and which are separated by the chordal ring domain $U_{m_{1}}$. We consider all the effective sets in $\Theta_{m_{2}-2}$ and repeat step (1). If there are no two effective sets in $\Theta_{m_{2}-2}$, which are close relative sets to each other, and which are separated by the chordal ring domain $U_{m_{1}}$, we consider all the effective sets in $\Theta_{m_{2}-3}$, and so on. Since the chordal ring domain $U_{m_{1}}$ separates $\Delta_{m_{0}}^{\prime} \cap L(G)$, after a finite number of repeating steps we will come to the situation in the first step. 
There are two effective sets of some set $\Theta_{j}$ for $m_{0} \leq j \leq m_{2}-1$ which are close relative sets to each other such that these two effective sets are separated by the chordal ring domain $U_{m_{1}}$. The remaining proof is exactly the same as for step (1). This shows that there are always contradictions with the assumption that the limit set $L(G)$ is not uniformly perfect. This completes the proof of Theorem 1.1.

\section{SChotTKY-TyPe QUASICONFORMAL GROUPS}

In this section, we deal with Schottky-type quasiconformal groups. We show that the limit set of a given Schottky-type quasiconformal group is uniformly perfect, and this generalizes our main result in the last section. Then we show that if a given discrete quasiconformal group contains two loxodromic elements with disjoint fixed point sets, then the limit set of the quasiconformal group has positive Hausdorff dimension. Further we prove that discrete non-elementary quasiconformal groups have limit sets with positive Hausdorff dimension.

For $t \geq 2$ let $D_{1}, D_{2}, \ldots, D_{2 t-1}, D_{2 t}$ be $2 t$ continua with non-empty interiors and disjoint closures. We suppose that there are $t$ quasiconformal mappings $g_{1}, g_{2}, \ldots, g_{t}$ such that

$$
g_{i}\left(\stackrel{\circ}{D}_{2 i-1}\right)=\overline{\mathbb{R}}^{n} \backslash \bar{D}_{2 i},
$$

where $\stackrel{\circ}{D}_{2 i-1}$ is the interior of the set $D_{2 i-1}$.

Let $G=\left\langle g_{1}, g_{2}, \ldots, g_{t}\right\rangle$ be the group generated by the quasiconformal mappings $g_{1}, g_{2}, \ldots, g_{t}$. We say that the group $G$ is a Schottky-type quasiconformal group if $G$ is a quasiconformal group.

5.1. Lemma. Let $G=\left\langle g_{1}, g_{2}, \ldots, g_{t}\right\rangle$ be a Schottky-type quasiconformal group. Then the group $G$ is a discontinuous group, and hence it is a discrete group.

5.2. Lemma. Let $G=\left\langle g_{1}, g_{2}, \ldots, g_{t}\right\rangle$ be a Schottky-type quasiconformal group. Then the limit set $L(G)$ of the group $G$ is a perfect set.

Proof. The group $G$ is a quasiconformal group, and thus it is a convergence group. By Lemma 5.1 the group $G$ is a discrete convergence group. Also note that the limit set $L(G)$ contains more than three points. Therefore the limit set $L(G)$ is a perfect set.

Let $A_{1}$ be the closure of the union of all the sets $D_{1}, D_{2}, \ldots, D_{2 t-1}$ and $D_{2 t}$. First let us consider the mappings $g_{1}$ and $g_{1}^{-1}$. The images of $D_{2}, D_{3}, \ldots, D_{2 t-1}$ and $D_{2 t}$ under the mapping $g_{1}$ are all inside the set $D_{2}$. The images of all the sets $D_{1}, D_{3}, \ldots, D_{2 t-1}$ and $D_{2 t}$ under $g_{1}^{-1}$ are all inside the set $D_{1}$. Similarly, for any $i$ with $2 \leq i \leq t$, we consider the mappings $g_{i}$ and $g_{i}^{-1}$. The images of $D_{1}, D_{2}, \ldots$, $D_{2 t-1}, D_{2 t}$ under the mapping $g_{i}$ are all inside the set $D_{2 i}$, and the images of $D_{1}, D_{2}, \ldots, D_{2 i-1}, D_{2 i+1}, \ldots, D_{2 t-1}, D_{2 t}$ under the mapping $g_{i}^{-1}$ are all inside the set $D_{2 i-1}$. We call all these image sets the second level sets of the group $G$. We denote by $A_{2}$ the closure of the union of all the second level sets of $G$. In general, we can define $A_{p}$ to be the closure of the union of all the $p$-th level sets of the group $G$.

For $1 \leq p<\infty$, set

$$
D_{p}=\overline{\mathbb{R}}^{n} \backslash A_{p}
$$


the complement of $A_{p}$ with respect to $\overline{\mathbb{R}}^{n}$. Clearly $A_{p}$ is closed and $D_{p}$ is open. In addition,

$$
\begin{aligned}
& A_{1} \supset A_{2} \supset \ldots \supset A_{p} \supset \ldots, \\
& D_{1} \subset D_{2} \subset \ldots \subset D_{p} \subset \ldots
\end{aligned}
$$

5.3. Lemma. For $1 \leq p<\infty$, let $r_{p}$ be the maximum of the diameters of all the components of $A_{p}$. Then the limit of the sequence $\left\{r_{p}\right\}$ exists, and

$$
\lim _{p \rightarrow \infty} r_{p}=0 .
$$

5.4. Lemma. Let $G=\left\langle g_{1}, g_{2}, \ldots, g_{t}\right\rangle$ be a Schottky-type quasiconformal group of $\overline{\mathbb{R}}^{n}$. Then the limit set $L(G)$ of the group $G$ is given by

$$
L(G)=\bigcap_{p=1}^{\infty} A_{p} .
$$

5.5. Theorem. Let $G=\left\langle g_{1}, g_{2}, \ldots, g_{t}\right\rangle$ be a Schottky-type quasiconformal group of $\overline{\mathbb{R}}^{n}$. Then the limit set $L(G)$ of the group $G$ is uniformly perfect.

The proofs for the above two lemmas and the last theorem are exactly the same as for the Schottky quasiconformal group case. We omit the proofs for the lemmas and the theorem.

Next we consider the general discrete quasiconformal groups and prove the following theorem.

5.6. Theorem. Let $G$ be a discrete quasiconformal group. If there are two loxodromic elements $g_{1}$ and $g_{2}$ in the group $G$ such that

$$
\text { fix }\left(g_{1}\right) \cap \operatorname{fix}\left(g_{2}\right)=\varnothing,
$$

where fix $\left(g_{1}\right)$ and fix $\left(g_{2}\right)$ are fixed point sets of $g_{1}$ and $g_{2}$ respectively, then the Hausdorff dimension $\operatorname{dim}_{H} L(G)$ of the limit set $L(G)$ is positive.

Proof. Without loss of generality, we suppose that $x_{1}$ and $y_{1}$ are the repelling point and the attracting point of the loxodromic element $g_{1}$, respectively. Similarly, we use $x_{2}$ and $y_{2}$ to denote the repelling and attracting points of the mapping $g_{2}$, respectively. The four points $x_{1}, y_{1}, x_{2}, y_{2}$ are all distinct by assumption.

Now let $U_{1}$ and $U_{2}$ be neighborhoods of $x_{1}$ and $x_{2}$ respectively, and let $V_{1}$ and $V_{2}$ be neighborhoods of $y_{1}$ and $y_{2}$ respectively, and we can pick $U_{1}, U_{2}, V_{1}, V_{2}$ so that they are balls and have disjoint closures.

Note that

$$
\lim _{i \rightarrow \infty} g_{1}^{i}=y_{1}, \quad \lim _{i \rightarrow \infty} g_{1}^{-i}=x_{1}
$$

uniformly on compact subsets of $\overline{\mathbb{R}}^{n} \backslash\left\{x_{1}\right\}$ and $\overline{\mathbb{R}}^{n} \backslash\left\{y_{1}\right\}$ respectively, and

$$
\lim _{i \rightarrow \infty} g_{2}^{i}=y_{2}, \quad \lim _{i \rightarrow \infty} g_{2}^{-i}=x_{2}
$$

uniformly on compact subsets of $\overline{\mathbb{R}}^{n} \backslash\left\{x_{2}\right\}$ and $\overline{\mathbb{R}}^{n} \backslash\left\{y_{2}\right\}$ respectively. Thus we can choose an integer $k_{1} \geq 1$ such that

$$
g_{1}^{k_{1}}\left(\overline{\mathbb{R}}^{n} \backslash U_{1}\right) \subset V_{1},
$$

and similarly, we can pick an integer $k_{2} \geq 1$ such that

$$
g_{2}^{k_{2}}\left(\overline{\mathbb{R}}^{n} \backslash U_{2}\right) \subset V_{2} .
$$


Let $G_{1}=\left\langle g_{1}^{k_{1}}, g_{2}^{k_{2}}\right\rangle$ be the subgroup of $G$ generated by the elements $g_{1}^{k_{1}}$ and $g_{2}^{k_{2}}$. It is clear that the group $G_{1}$ is a Schottky-type quasiconformal group, and hence the limit set $L\left(G_{1}\right)$ is a uniformly perfect set. The Hausdorff dimension of the limit set $L\left(G_{1}\right)$ is positive. On the other hand,

$$
L(G) \supset L\left(G_{1}\right)
$$

and therefore we have

$$
\operatorname{dim}_{H} L(G) \geq \operatorname{dim}_{H} L\left(G_{1}\right)>0 .
$$

This completes our proof.

Proof of Theorem 1.2. By the Corollary 6.15 in [5], the group $G$ contains infinitely many loxodromic elements, no two of which have a common fixed point. We pick two loxodromic elements $g_{1}$ and $g_{2}$ of the group $G$ such that

$$
\text { fix }\left(g_{1}\right) \cap \operatorname{fix}\left(g_{2}\right)=\varnothing \text {. }
$$

By Theorem 5.6 we obtain

$$
\operatorname{dim}_{H} L(G)>0
$$

and this finishes our proof.

5.7. Corollary. Let $G$ be a discrete convergence group. If $G$ contains two quasiconformal mappings which generate a non-elementary quasiconformal group, then the Hausdorff dimension of the limit set $L(G)$ is positive.

5.8. Corollary. Let $G$ be a discrete non-elementary quasiconformal group. Then any closed non-empty $G$-invariant subset of $\overline{\mathbb{R}}^{n}$ has positive Hausdorff dimension.

Proof. Let $E$ be a closed non-empty $G$-invariant subset of $\overline{\mathbb{R}}^{n}$ and let $L(G)$ be the limit set of the group $G$. Then we have

$$
E \supset L(G) \text {, }
$$

since $L(G)$ is the smallest closed non-empty $G$-invariant subset of $\overline{\mathbb{R}}^{n}$. Hence we have

$$
\operatorname{dim}_{H} E \geq \operatorname{dim}_{H} L(G)>0
$$

and this completes our proof.

\section{ACKNOWLEDGEMENTS}

I thank my advisor Professor Aimo Hinkkanen and Dr. Porama Saengcharoenrat for their constant encouragement and support. Professor Hinkkanen suggested this problem and was extraordinarily helpful during the preparation of this paper.

\section{REFERENCES}

[1] A. F. Beardon and Ch. Pommerenke, The Poincaré metric of plane domains, J. London Math. Soc. (2) 18 (1978), 475-483. MR0518232 (80a:30020)

[2] Petra Bonfert-Taylor and Edward C. Taylor, Hausdorff dimension and limit sets of quasiconformal groups, Mich. Math. J. 49 (2001), 243-257. MR.1852301|(2002g:30018)

[3] Petra Bonfert-Taylor and Edward C. Taylor, The exponent of convergence and a theorem of Astala, Indiana Univ. Math. J. 51 (2002), 607-623. MR.1911047 (2003c:30014)

[4] Lester R. Ford, Automorphic functions, Chelsea Publishing Company, New York, 1972.

[5] F. W. Gehring and G. J. Martin, Discrete quasiconformal groups I, Proc. London Math. Soc. (3) $5 \mathbf{5}$ (1987), 331-358. MF 0896224 (88m:30057)

[6] Aimo Hinkkanen, Julia Sets of Rational Functions are Uniformly Perfect, Math. Proc. Camb. Phil. Soc. 113 (1993), 543-559. MR.1207519 (94b:58084) 
[7] P. Järvi and M. Vuorinen, Uniformly Perfect Sets and Quasiregular Mappings, J. London Math. Soc. (2) 54 (1996), 515-529. MR1413895 (98d:30031)

[8] R. Mañé and L. F. da Rocha, Julia sets are uniformly perfect, Proc. Amer. Math. Soc. 116 (1992), 251-257. MR $1106180(92 \mathrm{k}: 58229)$

[9] B. Maskit, Kleinian groups, Springer-Verlag, 1987. MR0959135 (90a:30132)

[10] Ch. Pommerenke, Uniformly Perfect Sets and the Poincaré Metric, Arch. Math. 32 (1979), 192-199. MF 0534933 (80j:30073)

[11] Pekka Tukia, Convergence Groups and Gromov's Metric Hyperbolic Spaces, New Zealand J. Math. 23 (1994), 157-187. MR1313451 (96c:30042)

[12] Jussi Väisälä, Lectures on n-Dimensional Quasiconformal Mappings, Springer-Verlag, 1971. MR 0454009 (56:12260)

[13] Matti Vuorinen, Conformal Geometry and Quasiregular Mappings, Springer-Verlag, 1988. MR0950174 (89k:30021)

Department of Mathematics, University of Illinois at Urbana-Champaign, 1409 West Green Street, Urbana, Illinois 61801

E-mail address: xli5@math.uiuc.edu

Current address: Division of Science \& Mathematics, University of Minnesota, Morris, 600 East 4th Street, Morris, Minnesota 56267

E-mail address: lixiao@umn.edu 